\title{
IDENTIFICACIÓN DE FLAVONOIDES EN CATÁFILAS EXTERIORES DE Allium cepa L.
}

\author{
IDENTIFICATION OF FLAVONOIDS FROM Allium cepa L. PEEL
}

\author{
${ }^{1}$ Dániza Mirtha Guerrero Alva; ${ }^{2}$ Pablo Bonilla Rivera
}

\begin{abstract}
RESUMEN
Empleando las catáfilas externas de cebolla (Allium cepa L.) de las variedades roja arequipeña de producción convencional y "perita" amarilla orgánica como recurso, se obtuvieron nueve estructuras de flavonoides: dos flavonoles 3',4',5,6,7,8hexametoxi-O-Azúcarflavonol y 4,5,7-trihidroxiflavonol conocido como kaempherol el cual es un potente antioxidante y siete flavonas: 3',4',5,8-tetrametoxi-ORflavona; 4',5,6,7-tetrametoxiflavona; 5,7, dimetoxi-6-O-Azúcarflavona; 5-hidroxi-6,7,8trimetoxiflavona: 4',5,7-trihidroxi-3',6,8-trimetoxi-flavona; 3',4',5',6-trimetoxi-7-O-Azúcarflavona; 3',4',5,6,7,8-hexametoxi3-O-Azúcarflavona; y 3,4,6,7,8-pentametoxi-flavona. Los extractos deshidratados fueron añadidos a leche fluida y queso fresco, con notable éxito.
\end{abstract}

Palabras Clave: catáfilas exteriores de Allium cepa L., flavonoides.

\section{ABSTRACT}

Using the external catafilas of onion (Allium cepa L.) of the varieties Arequipeña red onion, of conventional production, and organic yellow "perita" as resource nine structures of flavonoids were obtained two flavonols 3',4',5,6,7,8-hexamethoxy-OAzflavonol and 4',5,7-trihydroxyflavonol known as kaempherol that is a strong antioxidant and seven flavones: 3',4',5,8tetramethoxy-or-flavone; 4, 5,6,7-tetramethoxyflavone; 5,7,dimethoxy-6-O-Azflavone; 5-hidroxy-6,7,8-trimethoxyflavone: 4',5,7-trihydroxy-3',6,8-trimethoxy-flavone; 3',4',5',6-trimethoxy-7-O-Azflavone; 3',4',5,6,7,8-hexamethoxy-3-O-Azflavone; and $3^{\prime}, 4^{\prime}, 6,7,8$-pentamethoxy-flavone. Dried extracts were added to milk and fresh cheese with success.

Key Words: Allium cepa L. peel, flavonoids.

\section{INTRODUCCIÓN}

La producción agrícola de la cebolla tiene un rol de primera importancia como fuente de alimento y calidad de vida. Pero no debería soslayarse la contaminación que produce, tanto en el campo de cultivo como en los lugares de distribución y comercialización. Las capas que envuelven al fruto, conocidas como catáfilas o cáscaras, al finalizar el ciclo de cultivo y entrando en la etapa de post cosecha, suelen ser retiradas manualmente del resto del bulbo, constituyéndose en pérdidas para los productores al convertirse en residuos orgánicos que no se venden ni industrializan y que adicionalmente son fuente de contaminación. En esa cadena de comercialización la cebolla vuelve a ser sometida a clasificación, operación que incluye el retiro de más catáfilas exteriores con el propósito de mejorar la presentación del producto ante el consumidor, generando abundantes desperdicios que se queman, al igual que en las fincas de los productores de cebolla orgánica. No olvidemos además que la cebolla es un cultivo muy extendido, pues se cuenta con numerosas variedades con distinta adaptación a las diferencias climáticas; China sobresale en la producción de cebolla a nivel mundial, mientras que en América Latina los mayores productores son México, Argentina y Brasil. En Perú debe mencionase en este contexto las regiones de Arequipa, Junín e Ica, con cebolla convencional para el mercado local y de exportación, además de la orgánica. Por ello, en el presente estudio pretendemos restituir al residuo su calidad de recurso y plantear su utilización, determinando la presencia de polifenoles tales como los flavonoides que tienen propiedades (Griffiths, G et al., 2002) antioxidantes, anticancerígenas y antiinflamatorias, y son por consiguiente de importancia para la salud humana y de aplicación industrial en alimentos procesados.

\section{MATERIALES Y MÉTODOS}

Las muestras constituidas por catáfilas externas de Allium cepa $\mathrm{L}$. fueron colectadas de dos zonas agrícolas: de cultivares orgánicos de la variedad amarilla ("perita") procedente de Pachacamac (Lurín, Lima); y de cultivares convencionales de la variedad roja de Camaná (Arequipa). La investigación experimental se desarrolló en el laboratorio de Ciencias Farmacéuticas y Recursos Naturales de la 
Facultad de Farmacia y Bioquímica de la Universidad Nacional Mayor de San Marcos.

\section{Extractos:}

Las catáfilas recogidas se seleccionaron eliminando todo resto de material vegetal fresco o diferente a la muestra, además de tierra y paja. Se lavó y picó finamente los dos kilos del material para proceder a la extracción de los compuestos de interés en alcohol de $70^{\circ} \mathrm{GL}$ por 7 dias, con movimiento del macerado y en oscuridad. Cada muestra se trató por separado, en un envase de vidrio de color ámbar con tapa hermética. Seguidamente cada uno de los extractos filtrados se deshidrató a una temperatura no mayor a $40^{\circ} \mathrm{C}$.

\section{Marcha fitoquímica:}

Las Reacciones de color efectuadas fueron las siguientes:

a. Reacción de Shinoda.

b. Reacción con álcalis: extracto acuoso y $\mathrm{NaOH}$ al $5 \%$.

c. Reactivo de Dragendorf.

d. Reactivo de Mayer.

e. Reacción con gelatina al 1\%.

f. Reacción con solución acuosa o etanólica de $\mathrm{FeCl}_{3}$. g. Reacción con naftol y $\mathrm{H}_{2} \mathrm{SO}_{4}$

\section{Técnicas cromatográficas:}

\section{CCFA Cromatografia en capa fina analitica.}

Se realizó sobre sílica gel tipo $\mathrm{G}(5 \times 20 \mathrm{~cm})$ y $(10 \times 20$ $\mathrm{cm})$, con indicador de fluorescencia a $254 \mathrm{~nm}$, de la empresa Merck, para determinación cualitativa de polifenoles y flavonoides. La mezcla de elusión fue diclorometano/metanol $\mathrm{y}$, tricloruro férrico y amonio como reveladores.

\section{CCFP Cromatografía de capa fina a escala preparativa.}

Se utilizó placas $(10 \times 20 \mathrm{~cm})$ y $(20 \times 20 \mathrm{~cm})$ con sílica gel tipo $\mathrm{G}$ e indicador de fluorescencia a $254 \mathrm{~nm}$, empleando como eluyentes diclorometano y metanol.

\section{CCR Cromatografia en columna rápida.}

La muestra a resolver se mezcló con sílica gel 60 para columna, de 0,063 a $0,200 \mathrm{~mm}$ de diámetro de la empresa Merck, colocándola como cabeza de columna. En la parte inferior y sobre papel filtro se distribuyó aproximadamente 30 gramos de sílica gel 60. Los solventes empleados fueron cloroformo, metanol y agua bidestilada, que fueron añadidos uno por vez sobre la muestra cubierta de papel filtro, empleando vacio.

\section{Espectroscopía:}

\section{EUV Espectroscopia ultravioleta.}

Los espectros de ultravioleta se obtuvieron con etanol en una celda de cuarzo de $1 \mathrm{~cm}$ de lado para el análisis preliminar de la estructura de flavonoides, mediante absorción UV-Visible en un espectrómetro Hewler Packard $8452^{\mathrm{a}}$ de doble haz de luz, y un programa de lectura con estándares de flavonoides. Las lecturas se efectuaron a una longitud de onda entre 230 y $280 \mathrm{~nm}$ (banda II) y de 310 a $560 \mathrm{~nm}$ (banda I). Se incluyó los reactivos de desplazamiento siguientes: metóxido de sodio, cloruro de aluminio, ácido clorhidrico y acetato de sodio, para la elucidación de las estructuras.

Aplicación de los extractos de las catáfilas externas de cebolla (Allitum cepa L.)

Los flavonoides hallados en las catáfilas externas de las cebollas de procedencia orgánica y convencional fueron adicionados a leche y queso fresco, siendo evaluados por ocho jueces entrenados, mediante una escala hedónica (Wittig E., 2001), análisis de varianza y la prueba de Duncan correspondiente.

\section{RESULTADOS Y DISCUSIÓN}

\section{Obtención de los extractos totales}

Los extractos obtenidos de las catáfilas de cebolla amarilla fueron de color rojo naranja muy intenso mientras que el de las catáfilas externas de la variedad arequipeña, de color granate violáceo oscuro. Los rendimientos fueron: 87,88 gramos de extracto en polvo con las catáfilas orgánicas que equivale a 4,39\%; y 160,5 gramos con las catáfilas de la cebolla arequipeña que representó un $8,02 \%$.

\section{Marcha fitoquímica}

En los dos extractos se halló abundante presencia de polifenoles y flavonoides.

\section{Cromatografia de capa fina analítica}

La mejor mezcla de eluyentes en la variedad amarilla fue la de $6: 1$ y con la variedad arequipeña de 8:1. Las muestras no poseían fluorescencia y con los reveladores se comprobó la presencia de polifenoles y flavonoides.

\section{Cromatografía de columna rápida}

Con la prueba de solubilidad se eligió: diclorometano, metanol y agua bidestilada para efectuar la CCR. Las fracciones fueron deshidratadas a no más de $40^{\circ} \mathrm{C}$ y almacenadas en frascos de vidrio ámbar, herméticamente cerradas. Los rendimientos fueron: con la variedad amarilla orgánica de $3,33 \% ; 91,2 \%$ y $1,99 \%$; mientras que con las catáfilas rojas arequipeñas de $0,62 \% ; 81,6 \%$ y $5,29 \%$ respectivamente.

\section{Cromatografía de capa fina analítica}

La investigación se continuó con los extractos deshidratados de las fracciones solubles en metanol y agua bidestilada por CCR, de las catáfilas externas de Allium cepa L de la variedad de cebolla roja arequipeña y del cultivar orgánico. La mejor separación de los componentes de cada uno de los extractos eluidos en la mezcla diclorometano/metanol fue: en la variedad roja arequipeña, de 9:1 en la fracción soluble en metanol y de 7:1 en la fracción soluble en agua bidestilada. En la muestra amarilla orgánica, fue de 7:1 en la fracción soluble en metanol y de 9:1 en la fracción soluble en agua bidestilada. Las muestras no presentaron fluorescencia, pero sí polifenoles y flavonoides.

\section{Cromatografia de capa fina a escala preparativa}

En este paso se separaron 6 fracciones solubles en metanol y 5 fracciones solubles en agua bidestilada de la muestra de cebolla roja arequipeña procedente de la CCR; y 6 fracciones solubles en metanol y 5 fracciones solubles en 
agua bidestilada de la variedad amarilla orgánica, correspondiente a los productos de la CCR. También se halló el valor de Rf de cada fracción.

\section{Espectroscopía ultravioleta-visible}

Todos los espectros obtenidos se compararon con lo publicado por Mabry T. et al. (1970) y por el análisis de los espectros hallados con cada fracción empleando cada uno de los reactivos de desplazamiento y los patrones; se propone las estructuras químicas correspondientes únicamente a flavonoides (Mabry, T.. et al., 1970).

En las fracciones de Allium cepa L. variedad roja arequipeña provenientes de la CCR solubles en metanol de acuerdo al espectro en etanol de la fracción $\mathrm{C}_{1}$, esta muestra correspondería a un flavonol. Al tratarla con metóxido de sodio hubo marcado efecto batocrómico, que indicaría la presencia de hidroxilos libres en posición 4' y en 7, quedando confirmado al añadir acetato de sodio, dándose el efecto batocrómico; al hacer reaccionar con tricloruro de aluminio se observó efecto batocrómico y luego al tratarlo con $\mathrm{HCl}$ los picos se mantuvieron lo que indica presencia de hidroxilos libres en la posición 3 y 5 , así como ausencia de hidroxilos adyacentes o en posición orto. Por consiguiente se propone la siguiente estructura:<smiles>O=c1c(O)c(-c2ccc(O)cc2)oc2cc(O)cc(O)c12</smiles>

Figura $\mathrm{N}^{\circ} 01.4^{\prime}, 5$, 7-trihidroxiflavonol ó Kaempherol

En la fracción $\mathrm{C}_{2}$ el espectro en etanol indica que el compuesto es una flavona; al tratarla con metóxido de sodio hubo efecto batocrómico, lo que indica presencia de hidroxilos libres en posición 4' y en 7, confirmando con el acetato de sodio el efecto batocrómico observado; al hacer reaccionar con tricloruro de aluminio se observó efecto batocrómico lo que indica presencia de hidroxilos libres y al ser tratado con $\mathrm{HCl}$ se mantuvieron los picos, lo que indica la presencia de hidroxilo libre en posición 5 . Se propone la estructura siguiente:<smiles>COc1cc(-c2cc(=O)c3c(O)c(OC)c(O)c(OC)c3o2)ccc1O</smiles>

Figura $\mathrm{N}^{\circ} 02.4^{\prime}$, 5, 7-trihidroxi-3',6,8-trimetoxiflavona

En la fracción $\mathrm{C}_{3}$ el espectro en etanol indica la presencia de una flavona; al tratarla con metóxido de sodio se registró ligero efecto ipsocrómico, lo que indica que el hidroxilo en 7 está glicosidado y no existe hidroxilo libre en posición 4'; al hacer reaccionar con tricloruro de aluminio se observó ligero efecto ipsocrómico, confirmando la glicosidación y descomposición; al acidificar con $\mathrm{HCl}$ vemos que se regeneran los picos originales obtenidos al leer con etanol debido a que el $\mathrm{HCl}$ hidroliza parcialmente el azúcar, por lo que regresa a las lecturas originales, proponiéndose la estructura siguiente:<smiles>COc1ccc(-c2cc(=O)c3cc(OC)c(OC)cc3o2)cc1OC</smiles>

Figura $\mathrm{N}^{\circ} 03$. 3',4', 6- trimetoxi- 7- O-Azúcarflavona

En las fracciones $\mathrm{C}_{7}$ y $\mathrm{C}_{8}$ de Allium cepa $\mathrm{L}$. roja arequipeña solubles en agua bidestilada se evidenció las siguientes estructuras: en la fracción $\mathrm{C}_{7}$ el espectro en etanol indicó un flavonol glicosidado; tratado con metóxido de sodio se produjo descomposición, lo que indicaría que el compuesto no tiene hidroxilos libres; se confirma este resultado al hacerle reaccionar con tricloruro de aluminio al no observar cambio alguno, lo que indica ausencia de hidroxilos libres en la posición 3 y 5 , por no haber cambios batocrómicos, indicando ausencia de hidroxilos libres en la posición orto ya que no se registró cambios al añadir $\mathrm{HCl}$. Por estos hallazgos se propone la siguiente estructura:<smiles>COc1ccc(-c2oc3c(OC)c(OC)c(OC)c(OC)c3c(=O)c2OC(Cl)(Cl)Cl)cc1OC</smiles>

Figura $\mathrm{N}^{\circ} 04.3^{\prime}, 4^{\prime}$, 5, 6,7, 8-hexametoxi-3- O-Azúcar flavonol

En la fracción $\mathrm{C}_{8}$ se observó que el espectro en etanol corresponde a una flavona. $\mathrm{Al}$ tratarla con metóxido de sodio no hubo cambio en las lecturas, lo que indica ausencia de hidroxilos libres en posición $4^{\prime}$ y en 7 ; al hacer reaccionar con tricloruro de aluminio se mantienen los picos al igual que acidificando con $\mathrm{HCl}$, lo que indicaria ausencia de hidroxilos libres, por lo que se propone la estructura de la Figura $\mathrm{N}^{\circ} 05$.

En las fracciones de Allium cepa $\mathrm{L}$. variedad amarilla orgánica obtenidas por CCR solubles en metanol se identificaron las siguientes estructuras: 
<smiles>COc1ccc(-c2cc(=O)c3cc(OC)c(OC)c(OC)c3o2)cc1OC</smiles>

Figura $\mathrm{N}^{\circ} 05.5 \cdot 3^{\prime}, 4^{\prime}, 6,7,8$ - pentametoxiflavona

En la fracción $D_{\text {, el espectro en etanol }}$ corresponde al de una flavona; tratada con metóxido de sodio no se registró efecto batocrómico, lo que indicaría que el compuesto no tiene hidroxilos libres en posición 4' y en 7, confirmando esta caracteristica al ser tratarla con acetato de sodio pues no hubo efecto batocrómico y desaparece la primera señal por descomposición. Al reaccionar con tricloruro de aluminio no se observó efecto batocrómico y con $\mathrm{HCl}$ los picos se mantuvieron, por ausencia de hidroxilos libres en la posición 5, así como ausencia de hidroxilo adyacente o en posición orto. Por lo expuesto se propone la siguiente estructura:<smiles>[R6]c1cc(OC)c2c(=O)cc(-c3ccc(OC)c(OC)c3)oc2c1OC</smiles>

Figura $\mathrm{N}^{\circ} 06.3^{\prime}, 4^{\prime}$, 5, 8-tetrametoxi-OR flavona

En la fracción $\mathrm{D}_{2}$ el espectro en etanol indica una flavona. Al ser tratada con metóxido de sodio no hubo efecto batocrómico lo que indicaría ausencia de hidroxilos en la posición 4' y en 7; al reaccionar con tricloruro de aluminio no se registraron cambios de lectura en el espectro y con $\mathrm{HCl}$ se mantuvieron los picos, lo que indicaría que en posición 5 no hay hidroxilo libre, así como ausencia de hidroxilos adyacentes o en posición orto, por lo que se propone la estructura:<smiles>COc1ccc(-c2cc(=O)c3c(OC)c(OC)c(OC)cc3o2)cc1</smiles>

Figura $\mathrm{N}^{\circ} 07.4$ ', 5 ,6, 7-tetrametoxiflavona
Con la fracción $\mathrm{D}_{3}$ el espectro en etanol indica que el esqueleto básico corresponde a una flavona. Tratada con metóxido de sodio no hubo efecto ipsocrómico significativo lo que indicaría que el compuesto no tiene hidroxilos libres; al reaccionar con tricloruro de aluminio se observó efecto ipsocrómico, lo que indicaría la presencia de un hidroxilo glicosidado en la posición 6 , no habiendo hidroxilo libre en la posición 5 , pues no se observaron cambios batocrómicos, además indica ausencia de hidroxilos libres en posición orto, ya que al reaccionar con $\mathrm{HCl}$ no hubo modificaciones en la lectura del espectro; por tanto se propone la estructura siguiente:<smiles>COc1cc2oc(-c3ccccc3)cc(=O)c2c(OC)c1O[W]</smiles>

Figura $\mathrm{N}^{\circ} 08$. 5, 7-dimetoxi-6- O-Azúcar flavona

Fracción $\mathrm{D}_{11}$ de Allium cepa L. variedad amarilla orgánica soluble en agua bidestilada. De acuerdo al espectro en etanol el esqueleto básico corresponde a una flavona, al ser tratada con metóxido de sodio se produjo efecto batocrómico lo que indica la presencia de hidroxilos libres en la posición 4' y en 7, y queda comprobado al reaccionar con acetato de sodio por observarse efecto batocrómico, señal de la presencia de hidroxilos libres en las posiciones $7 \mathrm{y}$ 4'. Al hacer reaccionar con tricloruro de aluminio se produjo efecto batocrómico que se mantuvo al acidificar con $\mathrm{HCl}$, lo que indica que tiene un hidroxilo libre en la posición 5; proponiéndose la estructura:<smiles>COc1c(OC)c(O)c2c(=O)cc(-c3ccccc3)oc2c1OC</smiles>

Figura $\mathrm{N}^{\circ} 09$. 5-hidroxi-6,7,8-trimetoxiflavona

Las flavonas al igual que las isoflavonas, antocianidinas y flavonoles despiertan gran interés por su potencial anticancerígeno, especialmente en el tratamiento del cáncer de mama (Fink, B. et al., 2007) y por su valor nutricional (Sgroppo, S. et al., 2005).

Los flavonoides presentes en los extractos totales de Allium cepa $L$ de las variedades amarilla orgánica y roja arequipeña, añadidos a leche de vaca y queso fresco tuvieron excelente aceptación. 


\section{CONCLUSIONES}

Las catáfilas externas de Allium cepa $L$, son una fuente de polifenoles de tipo flavonoide que están presentes en las variedades amarillas o rojas de cultivo orgánico o convencional.

Se determinó por espectroscopía y reacciones de desplazamiento dos estructuras de flavonoles, una en la fracción soluble en metanol de la variedad de Allium cepa $L$, roja arequipeña, conocida en la literatura como kaempherol, que es un potente antioxidante y $3^{\prime}, 4^{\prime}, 5,6,7,8$-hexametoxi-3O-Azúcarflavonol en la fracción soluble en agua bidestilada de la variedad roja arequipeña.

Se determinó por espectroscopía y reacciones de desplazamiento siete estructuras correspondientes a flavonas. En la variedad amarilla orgánica soluble en metanol: 3',4',5,8-tetrametoxi-7-ORflavona; 4',5,6,7tetrametoxiflavona; 5,7-dimetoxi-6-O-Azúcarflavona; y una estructura en la fracción soluble en agua bidestilada: 5hidroxi-6,7,8-trimetoxiflavona. En la variedad roja arequipeña soluble en metanol se halló dos estructuras correspondientes a: 4',5,7-trihidroxi-3',6,8trimetoxiflavona; 3',4',6-trimetoxi-7-O-Azúcarflavona; y una estructura en la fracción soluble en agua bidestilada: $3^{\prime}, 4^{\prime}, 6,7,8$-pentametoxiflavona.
La aplicación de los extractos en leche tratada térmicamente y queso fresco fueron ampliamente aceptadas por el panel de evaluación sensorial.

\section{REFERENCIAS BIBLIOGRÁFICAS}

Fink B, Steck S, Wolff M, Britton J, Kabat G; Gauet M, Abrahamson P, Bell P, Schroeder J, et al. Dietary flavonoid intake and breast cancer survival among women on Long Island. Cancer epidemiology biomarkers and prevention 2007; $\operatorname{vol} 16,(11): 2285-92$.

Griffiths G, Trueman L,Crowther T, Thomas B, Smith B. Onion: a global benefit to health. Phythotherapy Res. 2002, vol 16:603-15.

Mabry T, Markham K, Thomas M. The Systematic Identification of Flavonoids, N. York, Springer-Verlag ed.; 1970.

Sgroppo S, Ancos B, Cano M, Avanza J. Modificaciones del contenido en quercetina en cebollas por acción de microondas. FACENA 2005; vol 21:93-102.

Wittig E. 2001. Evaluación Sensorial Una metodología actual para tecnología de alimentos.

http://mazinger.sisib.uchile.cl/repositorio/lb/ciencias_qu imicas_y_farmaceuticas/wittinge 01 /

\section{Correspondencia:}

Dániza Guerrero Alva: gdaniza $a$ hotmail.com
Fecha de Recepción: 02/06/2014

Fecha de Aceptación: 25/06/2014 